\title{
An essential role for the transcription factor HEB in thymocyte survival, Tcra rearrangement and the development of natural killer T cells
}

\author{
Louise M D'Cruz, Jamie Knell, Jessica K Fujimoto, and Ananda W Goldrath \\ University of California San Diego, Division of Biology, La Jolla, California, USA
}

\begin{abstract}
E proteins are basic helix-loop-helix transcription factors that regulate many key aspects of lymphocyte development. Thymocytes express multiple E proteins that are thought to provide cooperative and compensatory functions crucial for $\mathrm{T}$ cell differentiation. Contrary to that, we report here that the E protein HEB was uniquely required at the $\mathrm{CD} 4^{+} \mathrm{CD} 8^{+}$double-positive (DP) stage of $\mathrm{T}$ cell development. Thymocytes lacking HEB showed impaired survival, failed to make rearrangements of variable- $\alpha\left(V_{\alpha}\right)$ segments to distal joining- $\alpha\left(J_{\alpha}\right)$ segments in the gene encoding the $\mathrm{T}$ cell antigen receptor $\alpha$-chain (Tcra) and had a profound, intrinsic block in the development of invariant natural killer T cells ( $i \mathrm{NKT}$ cells) at their earliest progenitor stage. Thus, our results show that HEB is a specific and essential factor in T cell development and in the generation of the $i$ NKT cell lineage, defining a unique role for HEB in the regulation of lymphocyte maturation.
\end{abstract}

The E protein family of transcriptional regulators mediate lymphocyte differentiation by binding to DNA at E-box sites, controlling the expression of genes essential to lineage commitment, targeting antigen-receptor gene rearrangement and enforcing key developmental checkpoints ${ }^{1,2}$. In mammals, E proteins include E12 and E47 (E2A; splice variants encoded by $T c f 3$, called ' $T c f e 2 a$ ' here), HEB and E2-2, which in lymphocytes typically bind as homo- or heterodimers to consensus E-box sequences. The Id proteins are highly related but lack a DNA-binding domain and thus function to inhibit E protein activity when they are incorporated into E-Id heterodimers. E2A, HEB and E2-2 have all been linked to the regulation of thymocyte development. However, E2-2 functions mainly in the specification of precursors to the plasmacytoid dendritic cell lineage ${ }^{3}$. The combined activity of both E2A and HEB promote T cell antigen receptor (TCR) rearrangements and control the developmental progression, survival and proliferation of developing $\mathrm{T}$ cells, accounting for the majority of E protein function in the thymus ${ }^{2}$. E2A (E47) supports the expression of molecules involved in pre-TCR- and Notch-mediated signaling at the earliest stages of thymocyte development ${ }^{4}$ and directly regulates rearrangements of the gene encoding TCR $\beta(T c r b)^{5}$. Furthermore, E2A is needed to restrain $\mathrm{CD} 4^{-} \mathrm{CD} 8^{-}$double-

Correspondence should be addressed to A.W.G. (agoldrath@ucsd.edu).

Accession codes. UCSD-Nature Signaling Gateway (http://www.signaling-gateway.org): E2A; GEO: microarray data, GSE19923.

Note: Supplementary information is available on the Nature Immunology website.

AUTHOR CONTRIBUTIONS

L.M.D. designed the study, did experiments, analyzed data and wrote the manuscript; J.K. and J.K.F. did experiments and analyzed data; and A.W.G. designed the study, analyzed data and wrote the manuscript.

COMPETING INTERESTS STATEMENT

The authors declare no competing financial interests.

Reprints and permissions information is available online at http://npg.nature.com/reprintsandpermissions/. 
negative (DN) cells from proliferating or progressing to the double-positive (DP) stage until productive $T c r b$ rearrangement occurs ${ }^{1,6}$. Similarly, E proteins enforce the checkpoint for Tcra rearrangements, and the absence of the expression of both E2A and HEB by DP thymocytes results in the generation of CD8 single-positive (SP) T cells lacking rearranged TCR $\alpha$ chains and thus TCR expression ${ }^{7}$. In contrast to E2A deficiency, loss of HEB results in fewer thymocytes owing to a role for HEB in the transition from the DN stage to the DP stage $^{8}$, but this defect is partially overcome by the compensatory activity of $\mathrm{E}^{2} \mathrm{~A}^{8,9}$.

Although thymocytes lacking either E2A or HEB demonstrate notable perturbations in development, substantial numbers of $\mathrm{T}$ cells are nonetheless generated ${ }^{8,10}$. However, a block in thymocyte development is observed at the DN stage when a dominant negative mutation of HEB is introduced ${ }^{9}$ or both E2A and HEB are deleted at the DN stage ${ }^{11}$, which demonstrates that a minimum of $\mathrm{E}$ protein activity is necessary for $\mathrm{T}$ cell development. In the context of other lineages that require $\mathrm{E}$ proteins for specification, the effective replacement of $T c f e 2 a$, which encodes E2A, with $T c f 12$, which encodes HEB, suggests that the E proteins encoded by these genes function interchangeably in 'rescuing' B cell development and embryonic lethality ${ }^{12}$. Similarly, in neurogenesis, for which E proteins are also necessary, all of the $\mathrm{E}$ proteins are equally effective partners for neurogenic differentiation factor 2 , which suggests similar activity among the family members ${ }^{13}$. Thus, E2A and HEB have been thought to compensate for each other during lymphocyte development.

Natural killer T (NKT) cells rapidly produce many cytokines after activation, which affects the recruitment and function of cells that participate in subsequent innate and adaptive immune responses ${ }^{14-16}$. The majority of NKT cells express an invariant TCR $\alpha$ chain that mediates the recognition of glycolipid antigens presented by the major histocompatibility complex class I-like molecule CD1d (invariant NKT ( $i$ NKT) cells) ${ }^{14-16}$. Such glycolipids can be derived from microorganisms and mediate activation of $i$ NKT cells during infection, and the absence of $i$ NKT cells exacerbates pathology ${ }^{17}$. The $i$ NKT cells mature in the thymus, differentiating from DP thymocytes and proceeding through defined developmental stages at which their maturation is influenced by many signaling molecules, transcription factors and cytokines that are not essential for conventional T cells ${ }^{14}$. In mice, $i$ NKT cells can first be detected in the thymus as $\mathrm{CD} 24^{+} \mathrm{CD} 69^{+}$cells bearing the canonical variable $\alpha$ segment 14-to-joining $\alpha$-segment $18\left(\mathrm{~V}_{\alpha} 14-\mathrm{J}_{\alpha} 18\right)$ Tcra rearrangement after their positive selection mediated by interactions with signaling lymphocytic-activation molecule receptors and CD1d molecules expressed by other DP thymocytes. Thus, mutations that affect either signaling through these receptors or CD1d-mediated antigen presentation result in the absence of $i$ NKT cells at this early stage in development. As CD24 is downregulated, the cells proliferate and progress through three more developmental stages identified by the upregulation of CD44 and then NK1.1 (refs. ${ }^{14,18,19}$ ). Progression through the early expansion phase is dependent on the transcription factor $\mathrm{c}-\mathrm{Myc}^{20,21}$. During this maturation process, $i \mathrm{NKT}$ cells acquire the expression of molecules associated with $\mathrm{T}$ cell activation, NK cell receptors and the ability to produce cytokines and lyse target cells.

Many transcription factors have been linked to $i$ NKT maturation. The earliest stage of $i$ NKT cell development absolutely requires the transcription factor ROR $\gamma t$ to induce upregulation of the antiapoptotic molecule Bcl- $\mathrm{x}_{\mathrm{L}}$, which supports the survival of DP thymocytes ${ }^{22,23}$. The prolonged survival of DP thymocytes allows distal Tcra V-to-J rearrangements, to produce the canonical $i$ NKT TCR $\alpha$ chain ${ }^{22-24}$. Thus, $i$ NKT cell development is restored in ROR $\gamma \mathrm{t}$-deficient thymocytes by transgenic expression of Bcl- $\mathrm{x}_{\mathrm{L}}$ or a rearranged $\mathrm{V}_{\alpha} 14-\mathrm{J}_{\alpha} 18$ $\mathrm{TCR}^{22,23}$. DP thymocytes deficient for the transcription factor Runx 1 also fail to give rise to $i$ NKT cells; however, in this case the canonical rearrangements are detected, which suggests a requirement for differentiation or expansion ${ }^{23}$. The T-box transcription factor T-bet also 
affects $i$ NKT cell development, although at a later stage of thymic development ${ }^{25,26}$, probably by regulating interleukin 15 responsivenes ${ }^{27}$. The transcription factor Egr2 has also been found to be necessary for the generation of a normal $i$ NKT cell compartment, and Egr2 deficiency alters both proliferation and survival ${ }^{28}$. Notably, the promyelocytic leukemia zinc-finger transcription factor PLZF has been shown to regulate the acquisition of the characteristic activated phenotype and effector functions of $i$ NKT cells ${ }^{29,30}$. How these many transcription factors are coordinated to promote commitment to the $i$ NKT lineage and subsequent maturation is not well defined.

Although the role of $\mathrm{E}$ proteins in conventional $\mathrm{T}$ cell development has been studied extensively, how these important transcriptional regulators affect the development of $i$ NKT cells has not been investigated. Here we studied the development of $i$ NKT cells from DP thymocytes lacking HEB and/or E2A and made the unexpected finding that HEB 'instructs' a unique gene-expression program and regulates both survival and Tcra rearrangements in a manner distinct from E2A. Our results identify HEB as an essential regulator of thymocyte development and highlight previously unknown distinctions in the functions of $\mathrm{E}$ protein family members.

\section{RESULTS}

\section{E proteins are expressed during $\mathbb{N K T}$ cell development}

Although it is well established that E proteins are expressed by thymocytes and are necessary for the development of conventional $\alpha \beta \mathrm{T}$ cells ${ }^{2}$, it was unknown whether they are expressed by or function in thymocytes that commit to the $i$ NKT cell lineage. We initially examined whether E protein transcripts were expressed during $i$ NKT cell development. Many strategies have been used to characterize the developmental progression of $i$ NKT cells $^{2,5}$; for convenience, we used the following criteria to stage these subsets using $\alpha$ galactosylceramide-loaded CD1d tetramers (CD1d-tet) to identify cells bearing the canonical $\mathrm{V}_{\alpha} 14-\mathrm{J}_{\alpha} 18$ TCR rearrangement: CD1d-tet ${ }^{+} \mathrm{CD} 24^{+}$(stage 0), CD1dtet ${ }^{+} \mathrm{CD} 24^{\mathrm{lo}} \mathrm{CD} 44^{\mathrm{lo}} \mathrm{NK} 1.1^{-}$(stage 1 ), CD1d-tet ${ }^{+} \mathrm{CD} 24^{\mathrm{lo}} \mathrm{CD} 44^{\mathrm{hi}} \mathrm{NK} 1.1^{-}$(stage 2 ) and CD1dtet ${ }^{+} \mathrm{CD} 24^{\mathrm{lo}} \mathrm{CD} 44^{\mathrm{hi}} \mathrm{NK} 1.1^{+}$(stage 3 ). We sorted each subset of $i \mathrm{NKT}$ cells from wild-type thymocytes and compared their expression of E2A and HEB mRNA. Notably, both E2A and HEB transcripts were most highly expressed at stage 0 and were abruptly downregulated by stage 1 (Fig. 1a). HEB showed the most dynamic mRNA changes, with low expression at stage 1 and a progressive increase in expression during stages 2 and 3 (Fig. 1a), whereas E2A mRNA abundance remained low. Of note, E2-2 was expressed at stage 0; however, further analysis showed that E2-2 deficiency had no effect on $i$ NKT cell development (data not shown). These data demonstrated regulation of E-protein mRNA during development of $i$ NKT cells and suggested they could potentially affect the earliest commitment to the $i$ NKT cell lineage (stage 0$)$.

\section{HEB is essential for NKT cell development}

We next investigated the role of E proteins in $i \mathrm{NKT}$ cell development in mouse models of $\mathrm{T}$ lineage-specific deletion of $T c f e 2 a$ and/or $T c f 12$ in DP thymocytes. We crossed mice with loxP-flanked alleles of these genes $\left(T c f e 2 a^{\mathrm{f} / \mathrm{f}}\right.$ or $\left.T c f 12^{\mathrm{f} / \mathrm{f}}\right)$ with a mouse line transgenic for expression of Cre recombinase driven by the promoter of the mouse gene encoding CD4 $\left(C d 4-\mathrm{Cre}^{+}\right)^{7,11,31,32}$ to delete these genes specifically in DP thymocytes and thus subsequently maturing $\mathrm{T}$ cells. We generated the following mouse lines: $T c f e 2 a^{\mathrm{f} / \mathrm{f}} \mathrm{Cd} 4-\mathrm{Cre}^{+}$ (E2A-T cell knockout (E2A-TKO)), $T c f 12^{\mathrm{f} / \mathrm{f}} \mathrm{Cd} 4-\mathrm{Cre}^{+}$(HEB-T cell knockout (HEB-TKO)) and $T c f e 2 a^{\mathrm{f} / \mathrm{f}} T c f 12^{\mathrm{f} / \mathrm{f}} \mathrm{Cd} 4-\mathrm{Cre}{ }^{+}$(E2A- and HEB-T cell knockout (E2A-HEB-TKO)). Initially, we examined by flow cytometry conventional $\alpha \beta \mathrm{T}$ cell development in these lines with conditional gene deletion (Fig. 1b). Total thymocyte numbers and subset composition, 
determined by surface expression of CD4 and CD8, were similar in wild-type, E2A-TKO and HEB-TKO mice, whereas thymocyte development in E2A-HEB-TKO mice was considerably perturbed, with a higher frequency of the CD8 SP subset, as reported before ${ }^{7}$ (Fig. 1b). The number of thymocytes in additional developmental stages was unchanged in E2A-TKO and HEB-TKO mice relative to that in wild-type mice but was altered in E2AHEB-TKO mice (Supplementary Fig. 1). Thus, we observed no gross alterations in total cell number or thymic subsets due to single deficiency in E2A or HEB occurring at the DP stage, which indicated that each of these E proteins can compensate for the loss of the other at this stage.

We evaluated the $i$ NKT cell populations in thymus, liver and spleen identified by staining for CD1d-tet and TCR $\beta$ (Fig. 1c). Loss of E2A alone did not affect the $i$ NKT cell compartment at any stage (Fig. 1). In contrast, we found that a much lower frequency of $i$ NKT cells resulted from HEB ablation (Fig. 1). Furthermore, analysis of $i$ NKT cell numbers showed a similar paucity of $i$ NKT cells in both HEB-TKO and E2A-HEB-TKO mice (Fig. 1d). Closer analysis of mice with heterozygous deficiency in E protein suggested that loss of E2A may have affected frequency of $i$ NKT cells slightly, but this did not result in loss of overall $i$ NKT cell numbers (Supplementary Fig. 2a,b). To rule out the possibility that the unchanged $i \mathrm{NKT}$ cell compartment observed in E2A-TKO mice was due to incomplete deletion of the loxP-flanked Tcfe2a allele, we reconstituted wild-type mice with fetal liver cells from E2A-deficient or HEB-deficient embryos (germline deletion) and analyzed the $i$ NKT cell compartment. We observed normal frequencies of $i$ NKT cells in mice reconstituted with E2A-deficient cells, but a complete loss of $i$ NKT cells resulted in mice reconstituted with HEB-deficient cells (Supplementary Fig. 3).

To more accurately pinpoint the stage at which HEB was required during $i$ NKT cell maturation, we analyzed the developmental subsets on the basis of expression of CD24, CD44 and NK1.1 in the presence or absence of HEB or E2A or both, before and after enrichment for CD1d-tet ${ }^{+}$cells (Fig. 1e,f). This analysis showed a nearly complete loss of $i$ NKT cells in the absence of HEB at the earliest stage of development (stage 0), as well as in subsequent developmental stages (Fig. 1e,f), suggesting a block in development before stage 0 and the expansion phase. These data define a unique and essential role for HEB during $i$ NKT cell development, for which E2A is unable to compensate.

\section{The HEB-TKO INKT cell defect is cell intrinsic}

As $i$ NKT cells are positively selected by CD1d molecules expressed by cortical thymocytes $^{14,18,19}$ and the $C d 4$-Cre transgene induces deletion of the loxP-flanked Tcfe $2 a$ and Tcf 12 alleles in DP thymocytes, we wanted to address whether the phenotype observed for HEB-TKO cells was cell intrinsic or was due to a defect in the ability of the cortical thymocytes to select developing $i$ NKT cells. We generated mixed-bone marrow chimeras in which irradiated recipients were reconstituted with a 1:1 mixture of wild-type and HEBTKO donor bone marrow, each distinguishable by their congenic expression of CD45 alleles. After reconstitution, we enriched for CD1d-tet ${ }^{+}$thymocytes and determined the percentage of $i$ NKT cells in both the wild-type and HEB-TKO fractions (Fig. 2a). We observed that HEB-TKO donor cells were unable to generate CD1d-tet ${ }^{+} i$ NKT cells (even in the presence of wild-type cells) and that this defect was at the earliest stage of $i$ NKT cell development (as noted in HEB-TKO mice; Fig. 2a). Furthermore, HEB deficiency led to a loss of $i$ NKT cells at all other stages of development (stages 1,2 and 3) in the thymus and periphery even in the presence of wild-type thymocytes (Fig. 2b). Comparison of absolute numbers of $i$ NKT cells from these mixed chimeras confirmed that HEB deficiency did not solely affect frequency, as we observed extremely small numbers of $i$ NKT cells in the thymus, spleen and liver of HEB-TKO mice relative to the numbers in their wild-type counterparts (Fig. 2c). Mixed-bone marrow chimeras generated with wild-type and E2A- 
TKO, HEB-TKO donor cells showed similar results (Supplementary Fig. 4). We also examined expression of CD1d by wild-type and E protein-deficient lymphocytes and found no differences (Fig. 2d). Thus, we conclude that there is no evidence of a processing or presentation defect by cortical thymocytes, as wild-type cells were unable to 'rescue' the HEB-TKO $i$ NKT cell developmental defect.

\section{HEB regulates the survival and proliferation of DP thymocytes}

To address how HEB affects $i$ NKT cell selection from the DP population, we examined the survival and proliferation of thymocytes. Notably, we found that HEB deficiency at the DP stage accelerated cell death in vitro (Fig. 3a), which suggested a defect in survival. After 30 $\mathrm{h}$ in culture, $<30 \%$ of the HEB-TKO cells were negative for annexin $\mathrm{V}$, compared with $\sim 50 \%$ of the wild-type and E2A-TKO cells, and by $50 \mathrm{~h}$ only $\sim 5 \%$ of the HEB-TKO cells were still alive, compared with $\sim 35 \%$ of the wild-type and E2A-TKO cells (Fig. 3a). Similarly, E2A-deficient thymocytes (germline deletion) survived in vitro as well as wildtype and E2A-TKO cells did (Supplementary Fig. 5). Notably, we also found that HEBTKO DP thymocytes showed greater proliferation and DNA content than did their wild-type counterparts (Fig. 3b). Approximately twice as many HEB-TKO thymocytes as wild-type thymocytes incorporated 5-bromodeoxyu-ridine (BrdU) after a 12-hour pulse, and significantly more had $>2 \mathrm{~N}$ DNA content $(P=0.04)$, which indicated that they were in S-G2 phases of the cell cycle. Consistent with the results obtained with the DP population, the very few HEB-TKO CD1d-tet ${ }^{+}$cells incorporated more BrdU (Fig. 3c), which indicated that the failure to accumulate HEB-TKO $i$ NKT cells was not due to a defect in the proliferative burst after their positive selection. Thus, HEB deficiency affects both the survival and proliferation of developing thymocytes.

\section{HEB controls a unique gene-expression profile in thymocytes}

We next addressed whether HEB controlled a larger subset of genes distinct from the related E protein and frequent binding partner E2A. We compared the transcript profiles of wildtype, E2A-TKO, HEB-TKO and E2A-HEB-TKO DP thymocytes by microarray analysis. Loss of HEB at the DP stage affected the expression of many genes, with 215 genes upregulated more than 1.7-fold and 315 genes downregulated relative to their wild-type counterparts (Fig. 4a). In contrast, when we compared E2A-TKO and wild-type DP thymocytes, we found that a similar number of genes were upregulated by the E2A-TKO thymocytes (310 genes) but fewer were downregulated (31 genes; Fig. 4a), which suggested that HEB could compensate for many of the targets positively regulated by E2A when deletion occurred at the DP stage. Not unexpectedly, loss of both E2A and HEB resulted in more extensive changes in gene expression, with 541 genes upregulated and 407 genes downregulated compared with expression in their wild-type counterparts (Fig. 4a), which indicated that these two E proteins do act together in regulating many targets and that loss of both is not compensated for by the activity of other E proteins. Studies focusing on the HEB-regulated genes showed that E2A-HEB-TKO DP cells had a high correlation: $89 \%$ of the genes upregulated by HEB-TKO cells also descended to the left on a $P$ value-versusfold change 'volcano' plot, and $85 \%$ of the genes downregulated by HEB-TKO cells also descended to the right (Fig. 4b). Furthermore, those genes that were positively regulated by HEB did not show a similar pattern of expression in E2A-TKO cells, with only $52 \%$ also down-regulated (Fig. 4b), which indicated no correlation and supported the observation of a unique profile of genes regulated specifically by HEB. Notably, those genes upregulated because of HEB deficiency were similarly upregulated (84\%) by the E2A-TKO thymocytes (Fig. 4b).

In an additional analysis, we visualized the mean expression of a group of annotated genes whose transcripts were regulated differently by both HEB-TKO and E2A-HEB-TKO DP 
cells relative to wild-type DP cells (Fig. 4c) and defined clusters of genes that were regulated together after loss of HEB but not after loss of E2A. Notably, many molecules encoded by the genes upregulated after HEB deletion were involved in metabolic processes, including eleven ribosomal proteins and five that function in oxidative phosphorylation. We summarized changes in gene expression in HEB-TKO and E2A-HEB-TKO cells relative to the expression in wild-type DP cells and highlighted genes up- or downregulated in both (Fig. 4d). Many genes encoding cytokines and chemokines (and their receptors), such as $I l 17 r b$, Ill2rb, Il22, Ccr4 and Ccl19, as well as genes encoding molecules that influence survival and cell death, such as Rorc, Bcl2ll, Cox 7b, Cox7c and Bmf, showed similar trends. Thus, HEB specifically regulates a discrete subset of genes during thymocyte development, potentially affecting many biological pathways.

Using quantitative PCR, we confirmed some of the targets identified and also asked specifically whether HEB was required to support the expression of molecules known to be necessary for the development of $i$ NKT cells from DP cells. Transcripts of many genes encoding molecules known to regulate $i$ NKT cell development, including Sh2dla, Tox, Gata3, Runxl, Zbtb16, Myc and Slamf6, were not altered considerably in the absence of E2A or HEB or of both E2A and HEB (Supplementary Fig. 6). However, consistent with the microarray results, mRNA for ROR $\gamma \mathrm{t}$ and $\mathrm{Bcl}-\mathrm{x}_{\mathrm{L}}$ was less abundant in HEB-TKO DP cells than in wild-type DP cells (Fig. 4e). As ROR $\gamma$ t is known to promote Bcl- $\mathrm{x}_{\mathrm{L}}$ expression, and thus thymo-cyte survival, this finding provided a possible mechanism for the enhanced death of the HEB-TKO thymocytes. To determine whether HEB was potentially regulating ROR $\gamma \mathrm{t}$ directly, we did chromatin immunoprecipitation of wild-type thymocytes with an HEBspecific antibody and found that HEB was indeed bound to two E-box sites ${ }^{33}$ in the promoter of the gene encoding ROR $\gamma \mathrm{t}$ (Fig. 4f). This binding was lower in HEB-TKO cells (Supplementary Fig. 7) and was more prominent than E2A binding (Supplementary Fig. 7). Thus, we concluded that HEB regulates a unique gene-expression profile distinct from that of its sister protein E2A.

\section{Impairment of distal $\mathrm{J}_{\alpha}$ rearrangements in the absence of HEB}

During rearrangement of the $T c r a$ locus, the first rearrangements tend to use proximal $\mathrm{J}_{\alpha}$ genes, whereas distal $\mathrm{J}_{\alpha}$ gene segments are included during secondary rearrarangements ${ }^{34}$. The canonical $i$ NKT TCR rearrangement joins $\mathrm{V}_{\alpha} 14$ to the distal $\mathrm{J}_{\alpha} 18$ region and thus requires such secondary rearrangements and extended survival, which provides time for this to occur. We wanted to ascertain if the impaired survival of HEB-TKO thymocytes, mediated by lower expression of ROR $\gamma \mathrm{t}$ and $\mathrm{Bcl}-\mathrm{x}_{\mathrm{L}}$, resulted in fewer distal rearrangements by DP cells, which would in turn lead to fewer $i$ NKT cells and skewing of the TCR repertoire.

First, using quantitative PCR to detect $\mathrm{V}_{\alpha} 14$ or $\mathrm{V}_{\alpha} 3$ rearrangements to specific $\mathrm{J}$ segments, we found that HEB-TKO thymocytes and $\mathrm{CD}^{+}$and $\mathrm{CD} 8^{+}$splenic $\mathrm{T}$ cells showed a profound lack of rearrangements using distal J segments (Fig. 5a,b). This result was in contrast to results obtained with E2A-TKO cells, in which there was no difference in rearrangements compared with those of wild-type cells. Next we extracted genomic DNA from sorted wild-type or HEB-TKO DP thymocytes and $\mathrm{CD} 4^{+}$splenocytes and analyzed $\mathrm{V}_{\alpha} 14$ rearrangements with proximal $\mathrm{J}_{\alpha} 56$ and $\mathrm{J}_{\alpha} 48$ and distal $\mathrm{J}_{\alpha} 16$ and $\mathrm{J}_{\alpha} 9$ rearrangements (Fig. 5a). We analyzed $\mathrm{V}_{\alpha} 14-$ to- $_{\alpha}(\mathrm{x})$ rearrangements (where '( $\mathrm{x}$ )' is region $56,48,16$ or 9 ) by multiplex PCR and Southern blot analysis with a $\mathrm{V}_{\alpha} 14$-specific probe. We observed that $\mathrm{V}_{\alpha} 14-\mathrm{J}_{\alpha} 18$ rearrangements were largely absent (Fig. 5c), and if this rearrangement was detected in HEB-TKO DP samples, the efficiency was low (data not shown), in agreement with the quantitative PCR analysis. Notably, even more distal rearrangements, such as those to $\mathrm{J}_{\alpha} 16$ and $\mathrm{J}_{\alpha} 9$, were completely absent in HEB-TKO thymocytes and peripheral $\mathrm{T}$ cells (Fig. 5c). In contrast, when we examined $\mathrm{V}_{\alpha} 14-$ to- $_{\alpha}(\mathrm{x})$ gene rearrangements in the absence 
of E2A, we observed no differences in distal $\mathrm{J}_{\alpha}$ gene rearrangements. Finally, we also examined this issue by analyzing products of PCR-amplified $\mathrm{V}_{\alpha} 3$-to- $\mathrm{C}_{\alpha}$ (constant $\alpha$ segment) cDNA with probes specific for proximal and distal $\mathrm{J}$ segments ${ }^{34}$ and found loss of distal $\mathrm{J}$ segment use only among the HEB-TKO populations (Fig. 5d). Although greater proliferation of DP cells (Fig. 3) can result in degradation of the RAG-2 recombinase protein $^{35,36}$, which could in turn impair rearrangements ${ }^{37}$, we found that wild-type and HEB-TKO thymocytes expressed similar amounts of RAG-2 protein (Supplementary Fig. 8). Thus, HEB-TKO DP thymocytes and mature T cells show substantially impaired use of distal $\mathrm{J}_{\alpha}$ segments during rearrangement, consistent with their impaired survival and failure to generate $i \mathrm{NKT}$ cells.

\section{'Rescue' of HEB deficiency by expression of TCR or Bcl- $x_{L}$}

If the main role of HEB in $i$ NKT cell development is to promote distal Tcra rearrangements, we reasoned that provision of a rearranged $i$ NKT TCR $\mathrm{V}_{\alpha} 14-\mathrm{J}_{\alpha} 18$ transgene $\left(\mathrm{V}_{\alpha} 14 \mathrm{Tg}\right)$ would 'rescue' the $i$ NKT cell phenotype observed in the absence of HEB. Thus, we crossed $T c f 12^{\mathrm{f} / \mathrm{f}} \mathrm{Cd} 4-\mathrm{Cre}^{+}$mice with the $\mathrm{V}_{\alpha} 14 \mathrm{Tg}$ line and found that expression of the transgene supported significant $i$ NKT cell development, even in the absence of HEB (Fig. 6). The frequency and cell number of $i$ NKT cells in $\mathrm{V}_{\alpha} 14 \mathrm{Tg}^{+} \mathrm{HEB}-\mathrm{TKO}$ mice was largely restored in the thymus, liver and spleen compared with that of HEB-TKO mice (Fig. 6a,b), which suggested that HEB-mediated regulation of DP cell survival and Tcra rearrangements govern $i$ NKT cell maturation. We did observe a slight accumulation of cells at stage 1 (Fig. $6 \mathrm{c})$. Notably, we found that HEB transcripts were re-expressed as $i$ NKT cells progressed to stages 2 and 3 (Fig. 1), which suggested that HEB may influence these later stages of maturation as well. However, the rescued $i$ NKT cells that developed in the absence of HEB were both phenotypically and functionally mature in terms of downregulation of CD24, proliferation, expression of activation markers and ability to produce interferon- $\gamma$ after stimulation compared with wild-type cells (Supplementary Fig. 9). Similarly, we evaluated expression of the panel of $i$ NKT cell-specific genes and found that the $\mathrm{V}_{\alpha} 14 \mathrm{Tg}^{+} \mathrm{HEB}-\mathrm{TKO}$ $i$ NKT cells did not show altered abundance of any of the transcripts compared with that of their wild-type counterparts (data not shown). Thus, the rescue of $i$ NKT cells in the absence of HEB by expression of the canonical TCR $\alpha$ provides evidence that the defect originated from the failure of HEB-TKO thymocytes to survive and undergo successful distal rearrangements.

We reasoned that if HEB regulates ROR $\gamma$ t expression, then introduction of $\mathrm{Bcl}-\mathrm{x}_{\mathrm{L}}$, reported to be regulated by ROR $\gamma \mathrm{t}$ and to be essential for thymocyte survival, would restore $i \mathrm{NKT}$ cell development even in the absence of HEB. Using a retroviral vector expressing $\mathrm{Bcl}-\mathrm{x}_{\mathrm{L}}$, we infected HEB-TKO bone marrow stem cells and used these cells to reconstitute sublethally irradiated hosts. After reconstitution, we observed that the provision of Bcl- $\mathrm{x}_{\mathrm{L}}$ restored the development of $i$ NKT cells from HEB-TKO bone marrow, whereas empty vector did not (Fig. 6d). Furthermore, expression of Bcl- $\mathrm{x}_{\mathrm{L}}$ restored the survival and distal $\mathrm{J}_{\alpha}$ Tcra gene rearrangements of HEB-TKO thymocytes (Fig. 6d). These data provide support for the idea that HEB regulates thymocyte survival, Tcra rearrangements and $i$ NKT cell development through its control of the expression of ROR $\gamma t$ and Bcl- $x_{L}$.

\section{DISCUSSION}

Although E proteins are broadly recognized as important regulators of the development of conventional $\alpha \beta$ T cells, our study has highlighted the unexpected and specific roles of HEB at the DP thymocyte stage in the control of a unique gene-expression program, survival, Tcra rearrangement and the development of $i$ NKT-lineage cells. Notably, we found that HEB regulated the expression of a cohort of genes by DP cells independently of the related E protein E2A. HEB deficiency led to lower expression of ROR $\gamma \mathrm{t}$ and $\mathrm{Bcl}-\mathrm{x}_{\mathrm{L}}$, diminished 
survival and greater proliferation of DP thymocytes and specifically affected distal $\mathrm{J}_{\alpha}$ gene rearrangements of the Tcra locus, altering the entire TCR repertoire. Additionally, HEB was expressed early in $i$ NKT commitment and HEB deficiency abolished $i$ NKT cell development. Thus, our data define previously unappreciated roles for HEB during T cell development.

Published studies have shown that HEB acts as a heterodimer with E2A, influencing T cell development at both the DN and DP stages $7,9,11$. However, these blocks are incomplete, and substantial $\alpha \beta$ T cell development does occur in the absence of HEB, which supports the idea of compensatory roles for E2A and HEB. Furthermore, conditional deletion of both E2A and HEB in DP thymocytes leads to a failure of the DP-to-SP checkpoint for TCR expression, but normal $\mathrm{T}$ cell development was thought to proceed when only one $\mathrm{E}$ protein is deleted at this stage, which indicates that HEB and E2A also act together to control the

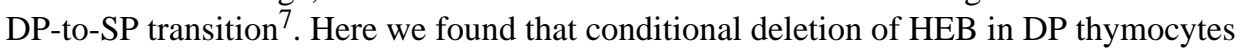
resulted in a nearly complete loss of differentiation of thymocytes to the $i$ NKT lineage but conditional deletion of E2A did not. This block was cell intrinsic and was not 'rescued' by wild-type thymocytes, which precludes the idea of a role for HEB in regulating proper processing and presentation of the CD1d-mediated positive selection. HEB-TKO thymocytes showed a block at the earliest detectable stage of $i$ NKT commitment, similar to ROR $\gamma \mathrm{t}-, \mathrm{Bcl}-\mathrm{x}_{\mathrm{L}}$ - and Runx1-deficient mice and apparently before Sap-, c-Myc-, PLZF- and Egr2-dependent $i$ NKT development ${ }^{20-23,28,29,38,39}$. The extreme paucity of cells at stage 0 and the greater proliferation of HEB-TKO DP cells and stage- 0 cells challenge the idea of an expansion defect, which occurs during the later transitions from stage 0 through stage 2 (refs. ${ }^{20,38}$ ).

The survival of HEB-TKO thymocytes was substantially impaired in vitro. Consistent with their impaired survival, HEB-TKO DP thymocytes had lower expression of ROR $\gamma \mathrm{t}$ and its target Bcl- $\mathrm{x}_{\mathrm{L}}$ and demonstrated greater proliferation, as do ROR $\gamma$ t-deficient thymocytes ${ }^{40}$. Notably, HEB directly binds E-box sites in the promoter regions of the gene encoding $\mathrm{ROR} \gamma \mathrm{t}$, which suggests direct regulation of ROR $\gamma \mathrm{t}^{33}$. ROR $\gamma \mathrm{t}$, through its regulation of Bcl$\mathrm{x}_{\mathrm{L}}$, is known to support prolonged survival and thus distal Tcra rearrangements ${ }^{33,34,41}$. The development of $i$ NKT cells is thought to be specifically impaired due to mutations that affect thymocyte survival, as the canonical Tcra rearrangement uses the $\mathrm{J}_{\alpha} 18$ segment, which is quite distal from the $V_{\alpha}$ gene segments and thus requires secondary rearrangements found only in cells that can survive long enough for this to occur. Consistent with that idea, HEB-TKO DP thymocytes demonstrated a profound defect in rearrangements using the $\mathrm{J}_{\alpha}$ segments most distal to the $\mathrm{V}$ segments regardless of whether these $\mathbf{J}_{\alpha}$ segments combined with $V_{\alpha} 14$ or with $V_{\alpha} 3$ gene segments. The failure to generate distal rearrangements also affected conventional $\mathrm{CD} 4^{+}$and $\mathrm{CD} 8^{+} \mathrm{T}$ cell populations, further emphasizing the role of HEB in influencing TCR repertoire. Notably, the expression of a rearranged $i$ NKT TCR transgene, which bypasses the need for prolonged survival at the DP stage for secondary rearrangements, resulted in considerable restoration of $i$ NKT cell development by HEBTKO thymocytes. We found that the $\mathrm{V}_{\alpha} 14 \mathrm{Tg}^{+} \mathrm{HEB}-\mathrm{TKO} i \mathrm{NKT}$ cells reached percentages and numbers near those of their $\mathrm{V}_{\alpha} 14 \mathrm{Tg}^{+}$wild-type control cells and were phenotypically and functionally mature. Likewise, the development, survival and distal Tcra rearrangements of HEB-TKO $i$ NKT cells were restored by Bcl- $x_{\mathrm{L}}$ expression, which provides further support for the idea that HEB-ROR $\gamma \mathrm{t}-\mathrm{Bcl}-\mathrm{x}_{\mathrm{L}}$ controls $\mathrm{T}$ cell development.

Our microarray gene-expression profiling studies showed that HEB uniquely controls expression of a substantial subset of genes at the DP stage. Among these targets are those encoding many cytokines, cytokine receptors, signaling molecules and regulators of metabolism and cell survival. Notably, loss of HEB did not alter transcript abundance in DP cells for many molecules that are key during iNKT cell development, including PLZF, c- 
Myc, Sap, Tox and Runx1. However, the DP defect resulting from HEB deficiency probably precedes the requirement for those molecules. We did observe moderately lower expression of Fyn and Slamf1 mRNA by HEB-TKO DP thymocytes, which indicates that signaling maybe affected. However, even complete loss of either Fyn or SlamF1 did not generate a block in $i$ NKT cell development as substantial as we observed after HEB deletion. Thus, our global gene-expression analysis of DP thymocytes has identified a subset of targets specifically regulated by HEB at this stage of development that are involved in a range of functions, which supports the idea of a unique role for HEB in T cell development. We have shown here that one specific role of HEB is to support thymocyte survival, allowing sustained Tcra rearrangements, affecting the total TCR repertoire and controlling $i$ NKT cell development. These observations fit into a broader view of E-protein function, whereby this family of transcription factors, which are expressed throughout hematopoiesis, is able to regulate specific aspects of lineage commitment, such as E2-2 in the development of plasmacytoid dendritic cells ${ }^{3}$, E2A in B cell commitment ${ }^{2}$ and now HEB in thymocyte survival and $i$ NKT cell development.

\section{METHODS}

Methods and any associated references are available in the online version of the paper at http://www.nature.com/natureimmunology/.

\section{Supplementary Material}

Refer to Web version on PubMed Central for supplementary material.

\section{Acknowledgments}

We thank C. Murre (University of California San Diego) for discussions, reagents and mice; Y. Zhuang (Duke University) for $T c f 12^{-I-}, T c f 12^{\mathrm{f} / \mathrm{f}}$ and $T c f e 2 a^{\mathrm{f} / \mathrm{f}}$ mouse lines; A. Bendelac (Howard Hughes Medical Institute) and M. Kronenberg (La Jolla Institute for Allergy and Immunology) for $\mathrm{V}_{\alpha} 14-\mathrm{J}_{\alpha} 18 \mathrm{TCR}$-transgenic mice; A. Abbas (University of California San Francisco) for the constructs MSCV2.2-Bcl-x $x_{L}-$ IRES-Thy-1.1 and MSCV2.2empty-IRES-Thy-1.1; M. Kronenberg for advice; I. Ch'en for advice and assistance; and J. Hamerman, G. Barton, E. Zuniga and S. Hedrick and members of the Goldrath laboratory for critical review of the manuscript. Supported by the Cancer Research Institute, the Pew Charitable Trusts, the National Institutes of Health (AI067545 and AI072117 to A.W.G.), the Leukemia \& Lymphoma Society (L.M.D.) and University of California San Diego undergraduate research scholarship program (J.K.F.).

\section{References}

1. Engel I, Johns C, Bain G, Rivera RR, Murre C. Early thymocyte development is regulated by modulation of E2A protein activity. J Exp Med 2001;194:733-745. [PubMed: 11560990]

2. Murre C. Helix-loop-helix proteins and lymphocyte development. Nat Immunol 2005;6:1079-1086. [PubMed: 16239924]

3. Cisse B, et al. Transcription factor E2-2 is an essential and specific regulator of plasmacytoid dendritic cell development. Cell 2008;135:37-48. [PubMed: 18854153]

4. Ikawa T, Kawamoto H, Goldrath AW, Murre C. E proteins and Notch signaling cooperate to promote T cell lineage specification and commitment. J Exp Med 2006;203:1329-1342. [PubMed: 16682500]

5. Rolf J, et al. Molecular profiling reveals distinct functional attributes of CD1d-restricted natural killer (NK) T cell subsets. Mol Immunol 2008;45:2607-2620. [PubMed: 18304639]

6. Engel I, Murre C. E2A proteins enforce a proliferation checkpoint in developing thymocytes. EMBO J 2004;23:202-211. [PubMed: 14685278]

7. Jones ME, Zhuang Y. Acquisition of a functional T cell receptor during T lymphocyte development is enforced by HEB and E2A transcription factors. Immunity 2007;27:860-870. [PubMed: 18093538] 
8. Barndt R, Dai MF, Zhuang Y. A novel role for HEB downstream or parallel to the pre-TCR signaling pathway during $\alpha \beta$ thymopoiesis. J Immunol 1999;163:3331-3343. [PubMed: 10477603]

9. Barndt RJ, Dai M, Zhuang Y. Functions of E2A-HEB heterodimers in T-cell development revealed by a dominant negative mutation of HEB. Mol Cell Biol 2000;20:6677-6685. [PubMed: 10958665]

10. Bain G, et al. E2A deficiency leads to abnormalities in $\alpha \beta$ T-cell development and to rapid development of T-cell lymphomas. Mol Cell Biol 1997;17:4782-4791. [PubMed: 9234734]

11. Wojciechowski J, Lai A, Kondo M, Zhuang Y. E2A and HEB are required to block thymocyte proliferation prior to pre-TCR expression. J Immunol 2007;178:5717-5726. [PubMed: 17442955]

12. Zhuang Y, Barndt RJ, Pan L, Kelley R, Dai M. Functional replacement of the mouse E2A gene with a human HEB cDNA. Mol Cell Biol 1998;18:3340-3349. [PubMed: 9584174]

13. Ravanpay AC, Olson JM. E protein dosage influences brain development more than family member identity. J Neurosci Res 2008;86:1472-1481. [PubMed: 18214987]

14. Godfrey DI, Berzins SP. Control points in NKT-cell development. Nat Rev Immunol 2007;7:505518. [PubMed: 17589542]

15. Geissmann F, et al. Intravascular immune surveillance by $\mathrm{CXCR}^{+}{ }^{+} \mathrm{NKT}$ cells patrolling liver sinusoids. PLoS Biol 2005;3:e113. [PubMed: 15799695]

16. Matsuda JL, Mallevaey T, Scott-Browne J, Gapin L. CD1d-restricted iNKT cells, the 'Swiss-Army knife' of the immune system. Curr Opin Immunol 2008;20:358-368. [PubMed: 18501573]

17. Tupin E, Kinjo Y, Kronenberg M. The unique role of natural killer T cells in the response to microorganisms. Nat Rev Microbiol 2007;5:405-417. [PubMed: 17487145]

18. Bendelac A, Savage PB, Teyton L. The biology of NKT cells. Annu Rev Immunol 2007;25:297336. [PubMed: 17150027]

19. Kronenberg M, Engel I. On the road: progress in finding the unique pathway of invariant NKT cell differentiation. Curr Opin Immunol 2007;19:186-193. [PubMed: 17303398]

20. Dose $\mathrm{M}$, et al. Intrathymic proliferation wave essential for $\mathrm{V} \alpha 14^{+}$natural killer $\mathrm{T}$ cell development depends on c-Myc. Proc Natl Acad Sci USA 2009;106:8641-8646. [PubMed: 19423665]

21. Mycko MP, et al. Selective requirement for c-Myc at an early stage of V $\alpha 14$ i NKT cell development. J Immunol 2009;182:4641-4648. [PubMed: 19342639]

22. Bezbradica JS, Hill T, Stanic AK, Van Kaer L, Joyce S. Commitment toward the natural T (iNKT) cell lineage occurs at the $\mathrm{CD} 4^{+} 8^{+}$stage of thymic ontogeny. Proc Natl Acad Sci USA 2005;102:5114-5119. [PubMed: 15792999]

23. Egawa T, et al. Genetic evidence supporting selection of the V $\alpha 14$ i NKT cell lineage from doublepositive thymocyte precursors. Immunity 2005;22:705-716. [PubMed: 15963785]

24. Hager E, Hawwari A, Matsuda JL, Krangel MS, Gapin L. Multiple constraints at the level of TCR $\alpha$ rearrangement impact V $\alpha 14$ i NKT cell development. J Immunol 2007;179:2228-2234. [PubMed: 17675483]

25. Matsuda JL, et al. T-bet concomitantly controls migration, survival, and effector functions during the development of Va14i NKT cells. Blood 2006;107:2797-2805. [PubMed: 16357323]

26. Townsend MJ, et al. T-bet regulates the terminal maturation and homeostasis of NK and $\mathrm{V} \alpha 14 \mathrm{i}$ NKT cells. Immunity 2004;20:477-494. [PubMed: 15084276]

27. Ohteki T, Ho S, Suzuki H, Mak TW, Ohashi PS. Role for IL-15/IL-15 receptor $\beta$-chain in natural killer $1.1^{+} \mathrm{T}$ cell receptor- $\alpha \beta^{+}$cell development. J Immunol 1997;159:5931-5935. [PubMed: 9550390]

28. Lazarevic V, et al. The gene encoding early growth response 2, a target of the transcription factor NFAT, is required for the development and maturation of natural killer T cells. Nat Immunol 2009;10:306-313. [PubMed: 19169262]

29. Kovalovsky D, et al. The BTB-zinc finger transcriptional regulator PLZF controls the development of invariant natural killer T cell effector functions. Nat Immunol 2008;9:1055-1064. [PubMed: 18660811]

30. Mattner J, et al. Liver autoimmunity triggered by microbial activation of natural killer T cells. Cell Host Microbe 2008;3:304-315. [PubMed: 18474357]

31. Pan L, Hanrahan J, Li J, Hale LP, Zhuang Y. An analysis of T cell intrinsic roles of E2A by conditional gene disruption in the thymus. J Immunol 2002;168:3923-3932. [PubMed: 11937548] 
32. Wolfer A, et al. Inactivation of Notch 1 in immature thymocytes does not perturb CD4 or CD8T cell development. Nat Immunol 2001;2:235-241. [PubMed: 11224523]

33. Xi H, Schwartz R, Engel I, Murre C, Kersh GJ. Interplay between ROR $\gamma$ t, Egr3, and E proteins controls proliferation in response to pre-TCR signals. Immunity 2006;24:813-826. [PubMed: 16782036]

34. Guo J, et al. Regulation of the TCR $\alpha$ repertoire by the survival window of CD ${ }^{+} \mathrm{CD} 8^{+}$thymocytes. Nat Immunol 2002;3:469-476. [PubMed: 11967541]

35. Li Z, Dordai DI, Lee J, Desiderio S. A conserved degradation signal regulates RAG-2 accumulation during cell division and links $\mathrm{V}(\mathrm{D}) \mathrm{J}$ recombination to the cell cycle. Immunity 1996;5:575-589. [PubMed: 8986717]

36. Lin WC, Desiderio S. Cell cycle regulation of V(D)J recombination-activating protein RAG-2. Proc Natl Acad Sci USA 1994;91:2733-2737. [PubMed: 8146183]

37. Yannoutsos N, et al. The role of recombination activating gene (RAG) reinduction in thymocyte development in vivo. J Exp Med 2001;194:471-480. [PubMed: 11514603]

38. Nichols KE, et al. Regulation of NKT cell development by SAP, the protein defective in XLP. Nat Med 2005;11:340-345. [PubMed: 15711562]

39. Savage AK, et al. The transcription factor PLZF directs the effector program of the NKT cell lineage. Immunity 2008;29:391-403. [PubMed: 18703361]

40. Benlagha K, Wei DG, Veiga J, Teyton L, Bendelac A. Characterization of the early stages of thymic NKT cell development. J Exp Med 2005;202:485-492. [PubMed: 16087715]

41. Sun Z, et al. Requirement for ROR $\gamma$ in thymocyte survival and lymphoid organ development. Science 2000;288:2369-2373. [PubMed: 10875923] 


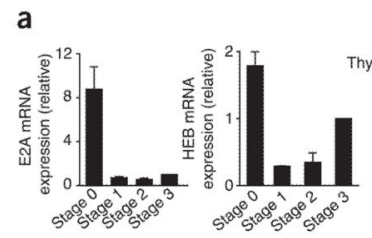

C

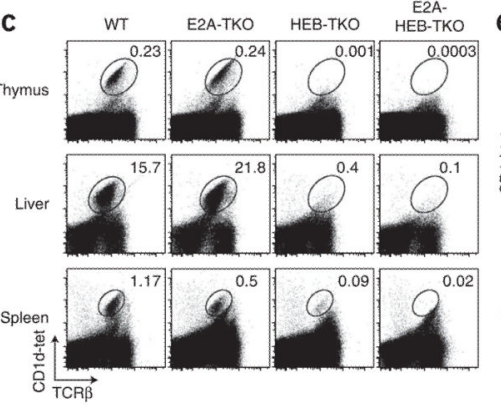

b

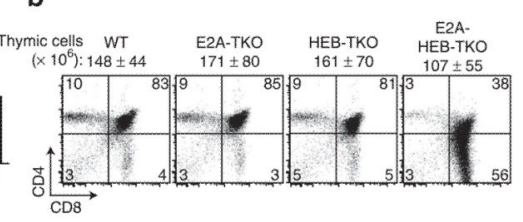

e

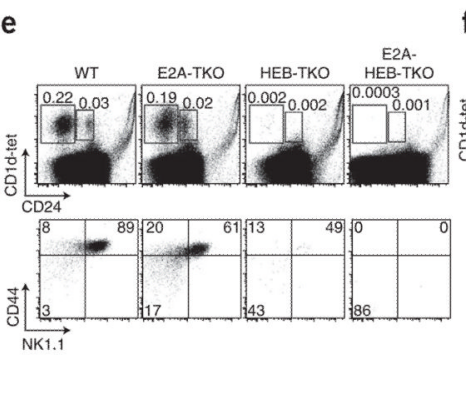

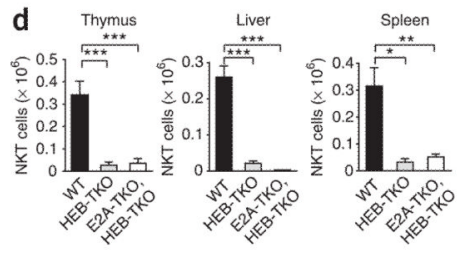

$\mathbf{f}$

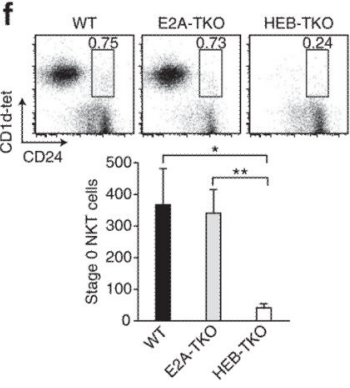

Figure 1.

HEB is essential for $i$ NKT cell development. (a) Expression of $T c f e 2 a(\mathrm{E} 2 \mathrm{~A}) \mathrm{mRNA}$ and $T c f 12$ (HEB) mRNA by sorted wild-type thymic CD1d-tet ${ }^{+} \mathrm{TCR}^{+}{ }^{+} \mathrm{NKT}$ cells, normalized to stage-1 expression. Data are representative of three independent experiments (average of two or three separate samples). (b) Flow cytometry of the surface expression of CD4 and CD8 by wild-type (WT), E2A-TKO, HEB-TKO and E2A-HEB-TKO thymocytes.

Thymocyte number above plots (average \pm s.d.); numbers in quadrants indicate percent cells in each. Data are representative of three experiments. (c) Expression of CD1d-tet and TCR $\beta$ by lymphocytes from thymus, liver and spleen. Numbers adjacent to outlined areas indicate percent CD1 ${ }^{+}$TCR $\beta^{+} i \mathrm{NKT}$ cells among lymphocytes. Data are representative of five independent experiments. (d) Total CD1d-tet ${ }^{+} \mathrm{TCR} \beta^{+} i \mathrm{NKT}$ cells in thymus, liver and spleen. Data are representative of seven experiments (average and s.e.m. of seven to nine mice per group). (e) Maturation subsets of thymocytes defined by expression of CD1d-tet and CD24 (top) or CD44 and NK1.1 (bottom) on CD1d-tet-gated $i$ KKT cells. Numbers adjacent to outlined areas or in quadrants indicate percent cells in each. Data are representative of three or more independent experiments. (f) Expression of CD1d-tet and CD24 by thymocytes after enrichment for CD1d-tet ${ }^{+}$cells by magnetic-activated cell sorting. Numbers adjacent to outlined areas indicate percent CD1d $\mathrm{d}^{+} \mathrm{CD} 24^{+} i \mathrm{NKT}$ cells. Below, total CD1d ${ }^{+} \mathrm{CD} 24^{+} i \mathrm{NKT}$ cells (average and s.e.m. of four to six mice per group). Data are representative of four experiments. $* P<0.05$, $* * P<0.005$, and $* * * P<0.0005$ (unpaired two-tailed $t$-test). 
a

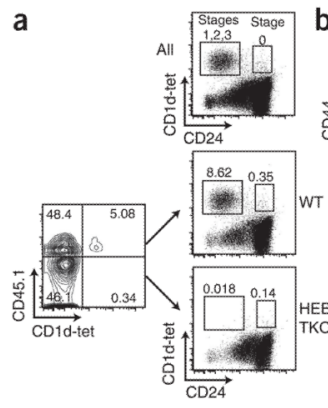

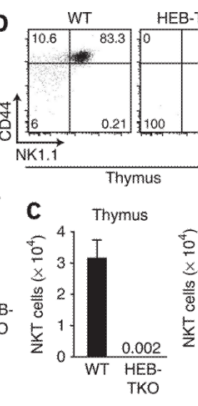

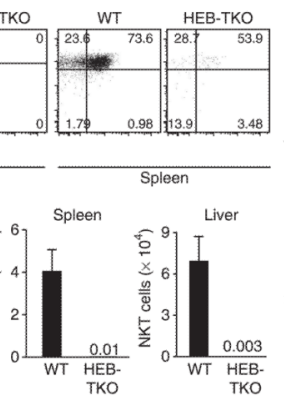

d
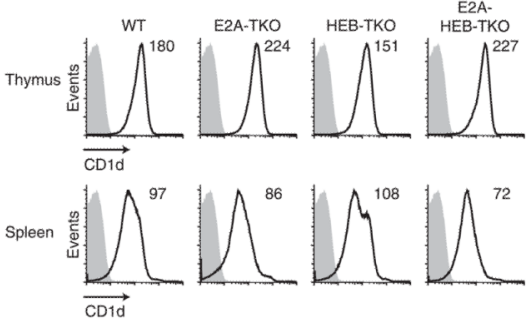

Figure 2.

The defect in $i$ NKT cell development by HEB-TKO thymocytes is cell intrinsic. (a) Expression of CD45.1 and CD1d-tet by donor thymocytes (left) and expression of CD1d-tet and CD24 by total thymocytes and wild-type (CD45.1 $1^{+}$) and HEB-TKO thymocytes in reconstituted recipients (right). Data are representative of two experiments. (b) Surface expression of NK1.1 and CD44 by CD1d-tet ${ }^{+}$donor cells from thymus and spleen of bone marrow chimeras. Data are representative of two experiments with five chimeras total. Numbers adjacent to outlined areas or in quadrants $(\mathbf{a}, \mathbf{b})$ indicate percent cells in each. (c) Total CD1d-tet ${ }^{+} \mathrm{TCR} \beta^{+}$cells among wild-type or HEB-TKO donor cell populations from thymus, spleen and liver. Numbers above HEB-TKO indicate average number of cells (bars not visible). Data are representative of QQ experiments (average and s.e.m. of five mice). (d) CD1d surface expression (black lines) by thymocytes and splenocytes from wild-type, E2A-TKO, HEB-TKO and E2A-HEB-TKO mice. Gray-filled histograms, unstained cells. Numbers in plots indicate mean fluorescence intensity. Data are representative of three independent experiments. 


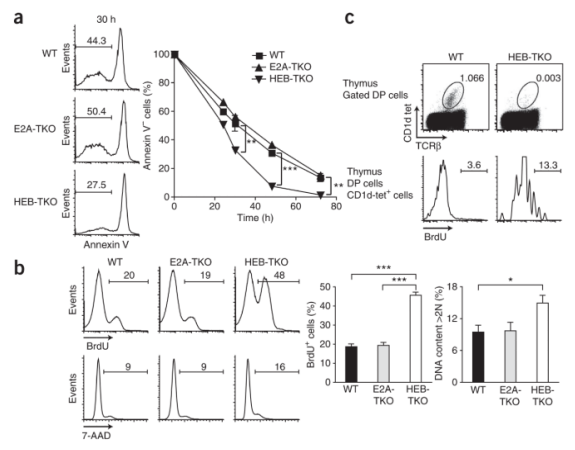

Figure 3.

HEB deficiency influences the survival and proliferation of thymocytes. (a) Annexin V expression by wild-type, E2A-TKO or HEB-TKO thymocytes cultured for 0-72 h. Numbers above bracketed lines (left) indicate annexin V-negative cells. Data are representative of five independent experiments. (b) BrdU incorporation and 7-amino-actinomycin D (7-AAD) staining of wild-type, E2A-TKO or HEB-TKO DP thymocytes after a 12-hour in vivo pulse of BrdU; numbers above bracketed lines indicate $\mathrm{BrdU}^{+}$cells (top) or $7-\mathrm{AAD}^{+}$cells (middle). Data are representative of four independent experiments (average and s.e.m.). (c) BrdU incorporation by wild-type or HEB-TKO CD1d-tet ${ }^{+}$DP thymocytes after a 3-hour pulse of BrdU. Numbers adjacent to outlined areas (top) indicate percent CD1d-tet ${ }^{+} \mathrm{TCR} \alpha^{+}$ cells; numbers above bracketed lines (bottom) indicate $\mathrm{BrdU}^{+}$cells. Data are representative of two independent experiments. $* P<0.05$, $* * P<0.005, * * * P<0.0005$ (unpaired twotailed $t$-test). 


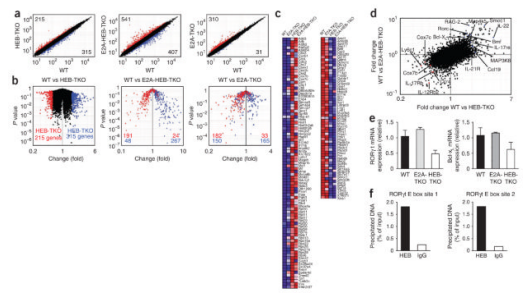

Figure 4.

Unique gene-expression profile of HEB-TKO DP cells. Affymetrix microarray analysis of mRNA from DP thymocytes sorted from wild-type, E2A-TKO, HEB-TKO and E2A-HEBTKO mice. (a) Normalized expression values for wild-type versus HEB-TKO (left), E2AHEB-TKO (middle) or E2A-TKO (right) DP thymocytes. Numbers in corners indicate number of genes with a difference in expression of 1.7-fold or more (upregulation, top left (red dots); downregulation, bottom right (blue dots)). (b) 'Volcano' plots of the geneexpression data of wild-type versus HEB-TKO (left), E2A-HEB-TKO (middle) or E2ATKO (right) DP cells. Numbers in plots indicate genes with a difference in expression of 1.7-fold or more in HEB-TKO DP cells versus wild-type cells (upregulation, red; downregulation, blue). Data are representative of experiments with two or three data sets per group. (c) Annotated gene expression of transcripts with a difference in expression of 1.7fold or more (upregulation, red; downregulation, blue). (d) Comparison of the geneexpression changes in wild-type versus E2A-HEB-TKO DP cells (horizontal axis) and wildtype versus E2A-HEB-TKO DP cells (vertical axis). Red and blue dots indicate transcripts uniquely up- and downregulated, respectively, by both HEB-TKO and E2A-HEB-TKO DP cells. (e) Expression of ROR $\gamma \mathrm{t}$ and Bcl- $\mathrm{x}_{\mathrm{L}}$ mRNA by E2A-TKO and HEB-TKO DP cells, normalized to the expression of GAPDH (glyceraldehyde phosphate dehydrogenase) and presented relative to their expression in wild-type cells. Data are representative of three experiments (average and s.e.m. of three or four individual samples). (f) Chromatin immunoprecipitation from wild-type thymocytes with antibody to HEB or control immunoglobulin $\mathrm{G}$ antibody (IgG), followed by quantitative PCR analysis of input or precipitated DNA containing ROR $\gamma \mathrm{t}$ E-box site 1 (left) or 2 (right). 


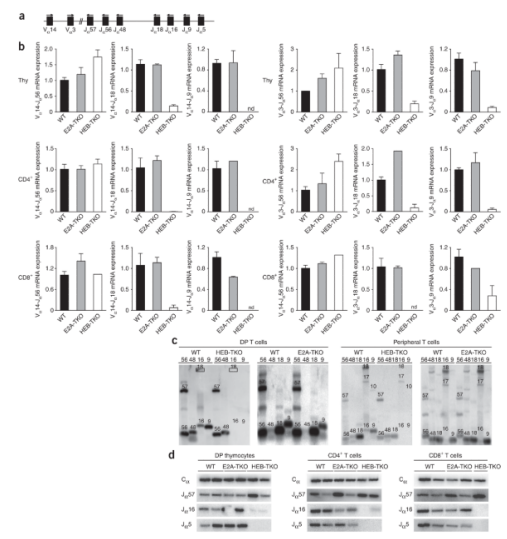

Figure 5.

Rearrangements of $\mathrm{V}_{\alpha}$ to distal $\mathrm{J}_{\alpha}$ segments are impaired in the absence of HEB. (a) TCR $\alpha$ locus; arrows above indicate primers used for PCR. (b) Quantitative PCR analysis of transcripts of $\mathrm{V}_{\alpha} 14$ and $\mathrm{V}_{\alpha} 3$ rearrangements to $\mathrm{J}_{\alpha} 56, \mathrm{~J}_{\alpha} 18$ or $\mathrm{J}_{\alpha} 9$ segments by sorted thymocytes (Thy; top) or $\mathrm{CD}^{+}$(middle) or $\mathrm{CD}^{+}$(bottom) splenocytes, presented relative to the expression of $\mathrm{C}_{\alpha}$ transcripts. Data are representative of two experiments (average and s.e.m. of two individual samples). (c) Southern blot analysis of multiplex PCR products of genomic DNA from sorted wild-type, HEB-TKO or E2A-TKO DP cells or CD4 ${ }^{+}$ splenocytes. DNA was amplified with $\mathrm{V}_{\alpha} 14$ forward primers and various $\mathrm{J}_{\alpha}(\mathrm{x})$ reverse primers (numbers above lanes indicate ' $\mathrm{x}$ '); numbers above bands indicate the amplified rearrangement (for example, '57' indicates $\mathrm{V}_{\alpha} 14-\mathrm{J}_{\alpha} 57$ ). Data are representative of three different experiments with three wild-type and HEB-TKO pairs, two wild-type and E2ATKO pairs for thymocytes and two per genotype for $\mathrm{CD}^{+}$cells. (d) RT-PCR analysis of $\mathrm{DP}, \mathrm{CD}^{+}$or $\mathrm{CD}^{+}$splenic $\mathrm{T}$ cells from pairs of wild-type, E2A-TKO or HEB-TKO mice with primers for $\mathrm{V}_{\alpha} 3-\mathrm{C}_{\alpha}$, followed by immunoblot analysis with a $\mathrm{J}_{\alpha}$-specific oligonucleotide probe. Top, internal $\mathrm{C}_{\alpha}$ oligonucleotide probe used for normalization of the template. Data are representative of one experiment. 

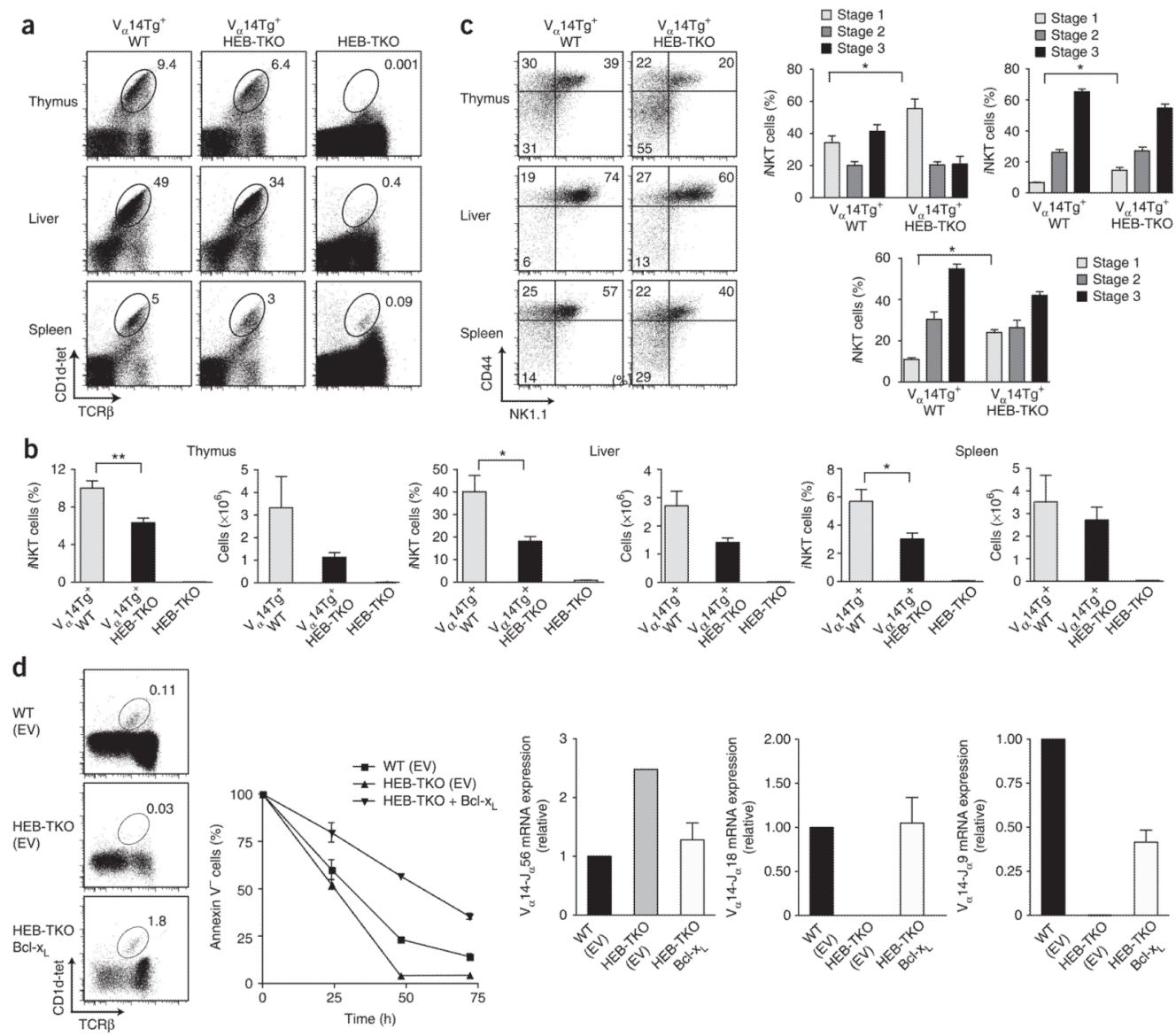

Figure 6.

Expression of a rearranged $\mathrm{V}_{\alpha} 14-\mathrm{J}_{\alpha} 18$ TCR transgene restores $i \mathrm{NKT}$ cell development in the absence of HEB. (a) Expression of CD1d-tet and TCR $\beta$ by $\mathrm{V}_{\alpha} 14 \mathrm{tg}^{+}$wild-type, $\mathrm{V}_{\alpha} 14 \mathrm{tg}^{+}$ HEB-TKO and HEB-TKO lymphocytes from thymus, liver and spleen. Numbers adjacent to outlined areas indicate percent CD1d-tet ${ }^{+} \mathrm{TCR} \beta^{+}$cells in gate. Data are representative of five independent experiments. (b) Frequency (left) and absolute number (right) of CD1dtet ${ }^{+} \mathrm{TCR} \beta^{+} i \mathrm{NKT}$ cells in thymus, liver and spleen of $\mathrm{V}_{\alpha} 14 \mathrm{tg}^{+}$wild-type, $\mathrm{V}_{\alpha} 14 \mathrm{tg}^{+} \mathrm{HEB}-$ TKO and HEB-TKO mice. Data are representative of two experiments (average and s.e.m.). (c) Surface expression of CD44 and NK1.1 by $i$ NKT cells in thymus, liver and spleen of $\mathrm{V}_{\alpha} 14 \mathrm{tg}^{+}$wild-type and $\mathrm{V}_{\alpha} 14 \mathrm{tg}^{+}$HEB-TKO mice (left); numbers in quadrants indicate percent cells in each. Right, frequency of $i$ NKT cells at developmental stages $1-3$. $* P<$ $0.05,{ }^{*} P<0.005,{ }^{* * *} P<0.0005$ (unpaired two-tailed $t$-test). Data are representative of three experiments (right, average and s.e.m. of four mice). (d) Expression of CD1d-tet and TCR $\beta$ (top left) by infected donor (CD45.2 $2^{+}$Thy- $1.1^{+}$) thymocytes in irradiated CD45.1 ${ }^{+}$ hosts reconstituted with wild-type or HEB-TKO bone marrow infected with empty vector (EV; control) or HEB-TKO bone marrow infected with retrovirus encoding $\mathrm{Bcl}-\mathrm{x}_{\mathrm{L}}$ (bottom). Right, annexin $\mathrm{V}$ expression by DP thymocytes obtained from the recipients described above and cultured for $0-72 \mathrm{~h}$. Below, quantitative PCR analysis of transcripts of $\mathrm{V}_{\alpha} 14$ rearrangements to $\mathrm{J}_{\alpha} 56, \mathrm{~J}_{\alpha} 18$ or $\mathrm{J}_{\alpha} 9$ segments by sorted thymocytes, presented relative to $\mathrm{C}_{\alpha}$ transcripts. Data are representative of two experiments (top left), two independent experiments (top right) or two experiments (below; average and s.e.m. of two individual samples). 